\title{
TERAHERTZ NDE FOR UNDER PAINT CORROSION DETECTION AND EVALUATION
}

\author{
Robert F. Anastasi ${ }^{1}$ and Eric I. Madaras ${ }^{2}$ \\ ${ }^{1}$ U.S. Army Research Laboratory, Vehicle Technology Directorate, AMSRD-ARL- \\ VT-SM, Nondestructive Evaluation Sciences Branch, NASA Langley Research \\ Center, Hampton, VA 23681 \\ ${ }^{2}$ NASA Langley Research Center, Nondestructive Evaluation Sciences Branch, \\ Hampton, VA 23681
}

\begin{abstract}
Corrosion under paint is not visible until it has caused paint to blister, crack, or chip. If corrosion is allowed to continue then structural problems may develop. Identifying corrosion before it becomes visible would minimize repairs and costs and potential structural problems. Terahertz NDE imaging under paint for corrosion is being examined as a method to inspect for corrosion by examining the terahertz response to paint thickness and to surface roughness.
\end{abstract}

Keywords: Terahertz NDE, Corrosion, Paint, Surface Roughness

PACS: $81.70 .-\mathrm{q}$

\section{INTRODUCTION}

Corrosion is a dissolving and wearing away of metal caused by a chemical reaction. In this electrochemical process, electrons in a system move from the anode to the cathode. The electron loss at the anode is called oxidation and metal loss occurs. At the cathode, electrons are captured and damage such as hydrogen embitterment can occur [1]. Since these corrosion byproducts may have nearly the same dielectric properties as the base material [2], inspecting for corrosion directly may not be feasible. However, the corrosion process generally affects a metallic surface with corrosion pits, cracking, or thinning. When this occurs under a layer of paint, the paint appears to swell and increase in thickness, and eventually crack and chip. In these cases, the corrosion changes a nominally smooth surface to an uneven and irregular surface that can be measured. Conventional nondestructive evaluation technologies (infrared, eddy current, ultrasonic, and radiography) have been used to inspect for under paint corrosion with the most promising being thermographic testing [3]. Near-field microwave techniques have also been used to detect for the presence of corrosion on an aluminum substrate under paint and primer [4].

QNDE 2005, 32 ${ }^{\text {nd }}$ Annual Review of Progress in Quantitative Nondestructive Evaluation, Bowdoin College, Brunswick, Maine, July 21 - August 4, 2005 
In the microwave measurements, dielectric properties of the paint, primer, and corrosion by-products were examined in the S-, G-, J-, X-, and Ku-Bands. The measurements showed that the dielectric properties of paint, primer, and aluminum oxide are very similar which reduce the detection of aluminum oxide directly. In addition, theses experiments demonstrate that higher frequencies with a standoff distance of a few $\mathrm{mm}$ were optimal for corrosion detection.

Terahertz $(\mathrm{THz})$ radiation occupies an area of the electromagnetic spectrum between the infrared and microwave bands and thus may prove useful for corrosion inspection. $\mathrm{THz}$ nondestructive evaluation is relatively new inspection technology $[5,6]$ that offers a non-contact and high-resolution means of inspecting for corrosion effects that may be hidden under a dielectric layer such as non-conductive paint. The frequency regime is between $100 \mathrm{GHz}$ and $10 \mathrm{THz}$ and has a free space wavelength of $300 \mu \mathrm{m}$ at $1 \mathrm{THz}$. This radiation, like microwaves, is able to penetrate and inspect nonconductive material for defects and is reflected from metallic materials. The radiation also reflects from interfaces that exhibit impedance discontinuities such as inclusions and voids. The advantage of $\mathrm{THz}$ over infrared techniques is that pulsed $\mathrm{THz}$ measures the electromagnetic time response that can be interpreted in a manner comparable to conventional ultrasonic time-domain signals. The advantage over microwave is the shorter wavelength given higher spatial and temporal resolution. Some other uses of $\mathrm{THz}$ have focused on spectroscopic applications such as inspecting the chemical content of packages [7], inspection of artwork and diagnostics of skin burn depth and severity [8], photonic crystal characterization [9], and response of $\mathrm{THz}$ to human skin [10]. In this paper, a $\mathrm{THz}$ system is used to examine the feasibility of inspecting for corrosion effects by examining variations in terahertz response due to changes in paint thickness and to surface roughness.

\section{MEASUREMENTS AND RESULTS}

The $\mathrm{THz}$ system used in this work was manufactured by Picometrix, Inc. THz signals are generated and detected using optical excitation of biased semiconductors with 70 -femto-second pulses from an $800 \mathrm{~nm}$ laser. The resulting digitized time-domain signal is composed of 2048 points and has a duration of 320 ps, which corresponds to a propagation path length in air of $96 \mathrm{~mm}$ or approximately 4 inches. The transmitter and receiver units were in a confocal arrangement and used a $15 \mathrm{~cm}$ diameter, $285 \mathrm{~mm}$ focal length lens to concentrate the THz energy on the sample. The spot size diameter on the sample was approximately $3 \mathrm{~mm}$. This arrangement is shown in Figure 1. This transmit/receive head was scanned in a raster pattern over an inspection sample with spatial increments of $1 \mathrm{~mm}$, while time-domain signals were digitized and stored for later processing.

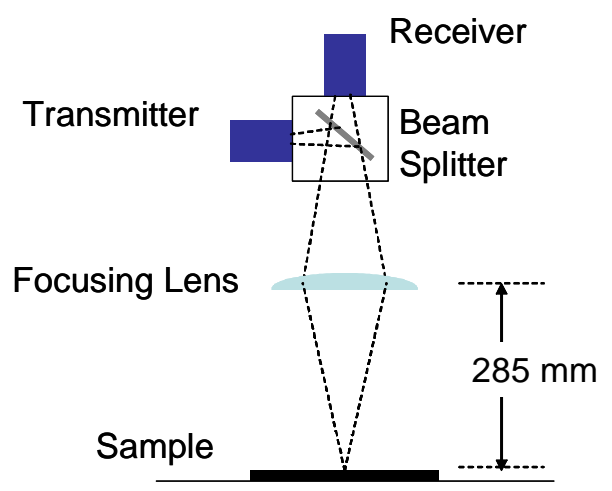

Figure 1. THz transmitter and receiver units in a confocal arrangement focused on the sample. 


\section{Paint Thickness}

$\mathrm{THz}$ response to paint thickness was examined using a sample with a varying thickness layer of paint on an aluminum substrate. An enamel spray paint was used to paint one primer layer and eight top coat layers each approximately $25 \mathrm{~mm}$ wide. The bar graph in figure 2 shows the range of paint layer thicknesses on the sample. Figure 3 shows the sample, figure $3 \mathrm{a}$, and $\mathrm{THz}$ signals at three different locations. Figure $3 \mathrm{~b}$ shows the signal for layer-9, paint thickness $0.335 \pm 0.0039 \mathrm{~mm}$. In this signal, the first peak of the paint surface echo and aluminum surface echo are resolved. Figure $3 \mathrm{c}$ shows the signal for an intermediate paint thickness, layer-6, paint thickness $0.183 \pm 0.0039 \mathrm{~mm}$, the individual echoes are not fully resolved in this signal and figure $3 \mathrm{~d}$, show a signal from the bare aluminum surface. Using the time between the paint surface echo and aluminum echo a wave velocity in the paint was found to be $0.190 \pm 0.013$ ps. This corresponds to a refractive index of $1.58 \pm 0.11$ and a dielectric constant of $2.50 \pm 0.36$.

A $\mathrm{THz}$ amplitude image is shown in figure 4. It was generated by plotting the maximum signal amplitude at each scan point as a function of gray-level. The image shows how the signal amplitude correlates to paint thickness. The darker gray or lower amplitude corresponds to thicker paint and the lighter gray, higher amplitude to thinner paint. The image also shows that paint thickness can be determined to a thickness of $\sim 0.050 \mathrm{~mm}$ even though the individual paint surface and aluminum substrate echoes could not be resolved. The influence of the color or paint pigment was not investigated.

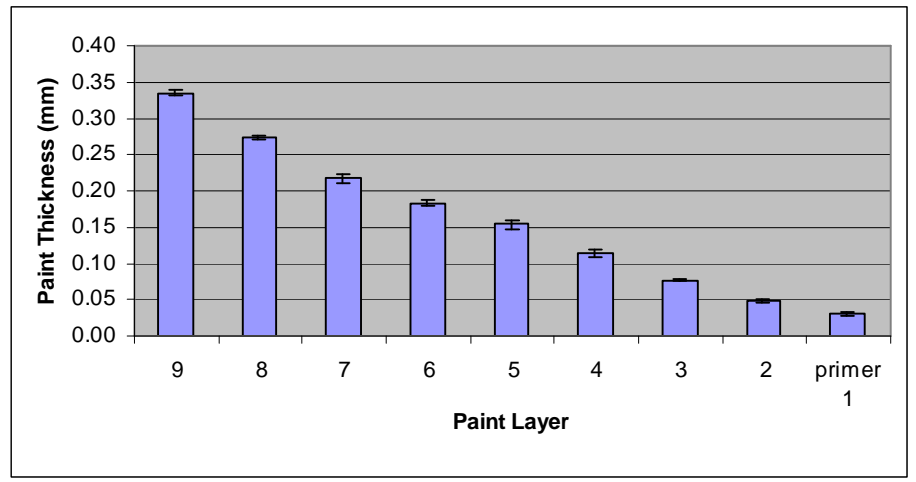

Figure 2. Paint layer thicknesses on an aluminum substrate.

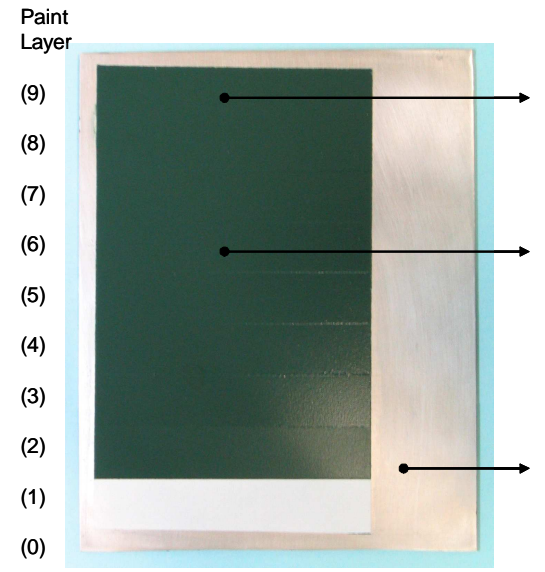

(a)
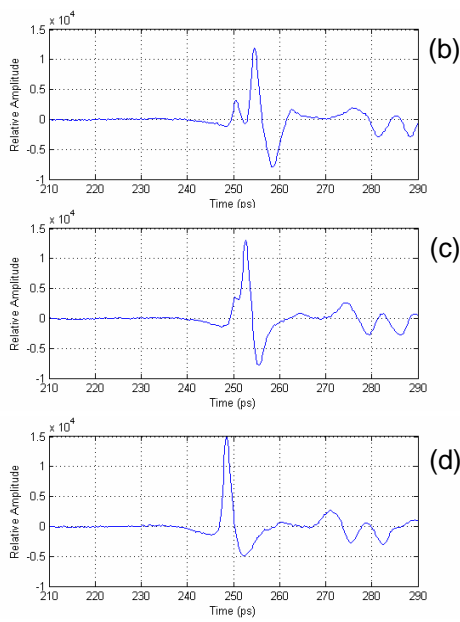

Figure 3. Paint sample (a) and (b) signal through layer-9, (c) through layer-6, and (d) on bare metal. 


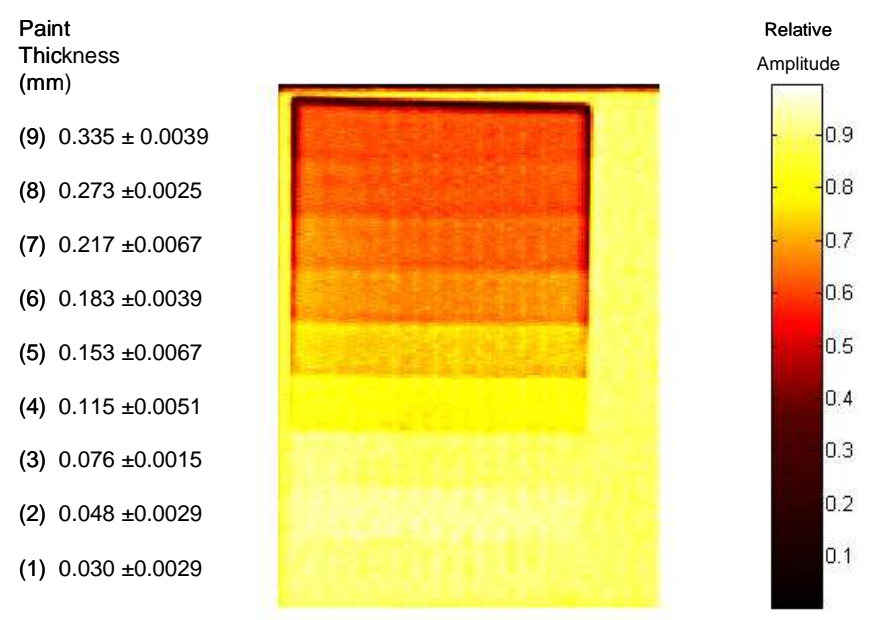

Figure 4. $\mathrm{THz}$ Amplitude Image (maximum signal amplitude).

\section{Surface Roughness}

Surface Roughness was examined using commercial comparator plates of various roughnesses. Four comparator plates used were standards for Planing and Shaping, Electrical Discharge Machining (EDM), Grit and Shot Blasting, and Casting. These plates covered a range of average roughness ( $\mathrm{Ra}$ ) from $0.04 \mu \mathrm{m}$ to $100 \mu \mathrm{m}$. The 'Planning and Shaping' plate is shown in figure 5a. This plate has eight separate areas of roughness. The smoothest area has an average roughness of $0.8 \mu \mathrm{m}$ and the roughest is $100 \mu \mathrm{m}$. The average roughness is the area between the roughness profile and its mean line, or the integral of the absolute value of the roughness profile height over the evaluation length [11]. These plates were inspected with the confocal $\mathrm{THz}$ head normal to the sample and at $5^{\circ}, 10^{\circ}, 15^{\circ}$ and $20^{\circ}$ from normal. The angle head arrangement was used to reduce the strong direct reflections from planar surfaces and examine if this arrangement improves surface roughness evaluation, figure $5 \mathrm{~b}$ show the $\mathrm{THz}$ head rotated $5^{\circ}$.

Amplitude and frequency images were generated from the $\mathrm{THz}$ scan data. The frequency image was generated by applying a Fast-Fourier-Transform (FFT) to each signal in the THz scan and then taking the mean amplitude of the spectrum between 0.4 and 0.6 $\mathrm{THz}$. For this data, this was the range of greatest spectral amplitude. The frequency images for $0^{\circ}, 10^{\circ}$, and $20^{\circ}$ are shown in figure 6 . These images illustrate that for a normal inspection, figure $6 \mathrm{a}$, the mean frequency amplitude is greater for the smooth areas than the roughest areas, thus the smooth areas reflect energy back to the detector while the rough areas scatter energy away from the detector. For the $10^{\circ}$ and $20^{\circ}$ angled inspection, figures $6 \mathrm{~b}$ and $6 \mathrm{c}$, the mean frequency amplitude is greater for the rough areas and less in the smooth areas, thus more $\mathrm{THz}$ energy is being detected for the rough area. To quantify the scatting shown in figure 6 , an average signal power per area was calculated for the four roughest areas on the 'Planing and Shaping' comparator plate. This was done by calculating an average signal for each roughness area and for the total inspection areas and then computing the average signal power for each area. The roughness area result was then divided by the total inspection area result. The outcome for the four areas is shown in figure 7. This shows that the roughest area and largest angle, backscatter had the most average-signal power. 


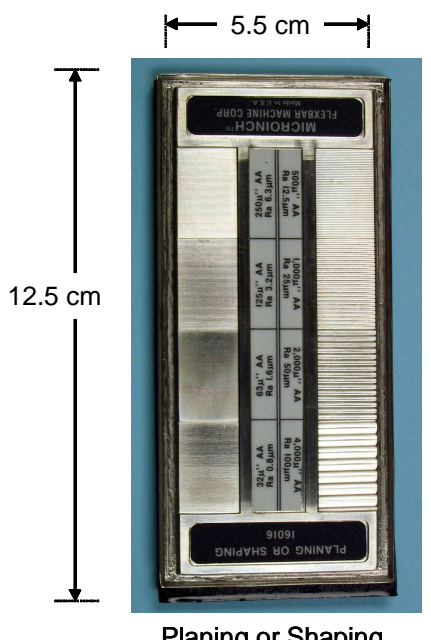

(a)

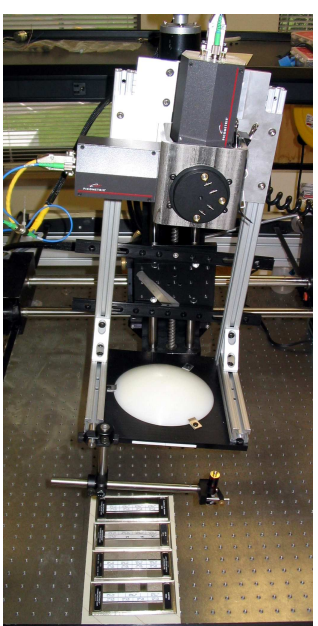

(b)

Figure 5. One of the comparator plate used in the surface roughness measurements (a) and the confocal head shown tilted at a 5 degree angle from normal.

(1)

(2)

(3)

(4)

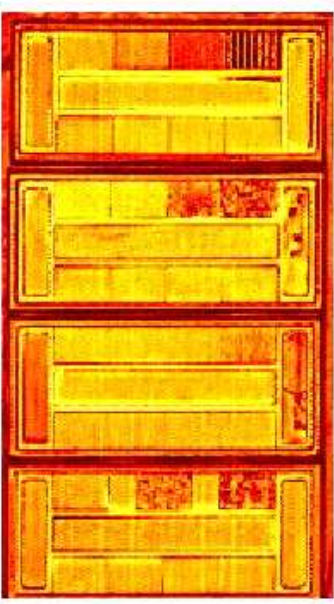

(a)

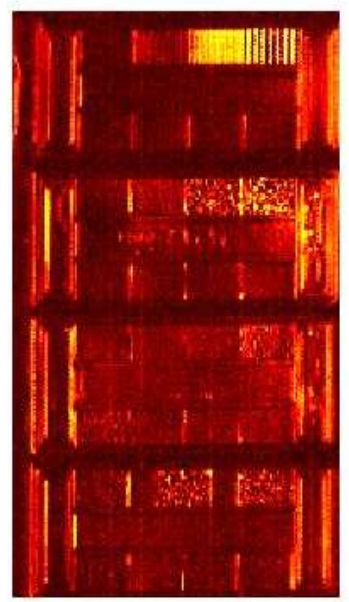

(b)

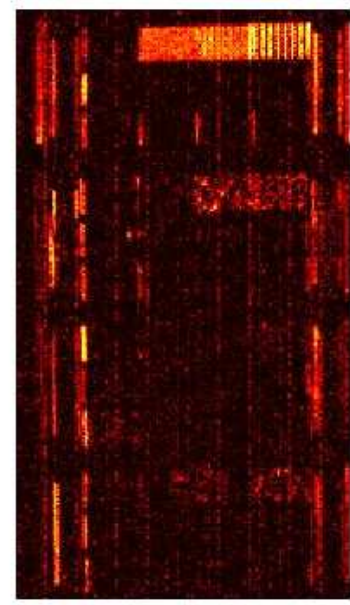

Relative Amplitude

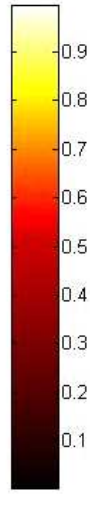

(c)

Figure 6. Comparator plate frequency images for the THz head at (a) $0 \mathrm{deg}$, (b) $10 \mathrm{deg}$, (c) 20 deg. Plate-1 is the Planning and Shaping, Plate-2 is EDM, Plate-3 is Grit and Shot Blasting, and Plate-4 is Casting.

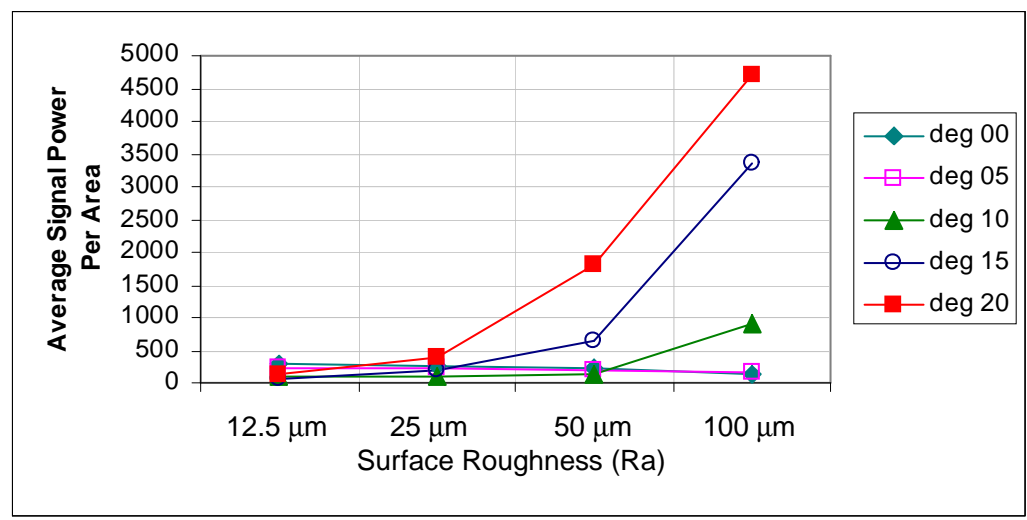

Figure 7. Average Signal Power per Area for four surface areas on the Planing and Shaping plate. 


\section{Corrosion Samples}

Two samples with corrosion were examined; a Fuselage Step Door from a Chinook Helicopter (courtesy of Ft. Eustis, United States Army Aviation Logistics School (USAALS), Newport News, VA) and a sample with manufactured corrosion (courtesy of Concurrent Technologies Corporation, Largo, FL). The step door was examined in normal and backscatter modes and the manufactured sample was examined in normal mode.

The Chinook fuselage step door, shown in figure 8, is approximately $20 \mathrm{~cm}$ by 14 $\mathrm{cm}$ and has areas of chipped, flaking and cracked paint. Two THz images are shown in figure 9. Figure 9a shows a maximum signal amplitude image. If the paint-thickness sample results are used as a reference then the areas of thick and thin paint can be identified by signal amplitude correlation, thus, the darker areas in the image maybe caused by thicker paint or potentially corrosion. Figure $9 \mathrm{~b}$, is a frequency image of mean amplitude between 0.62 and $0.68 \mathrm{THz}$, generated at a $10^{\circ}$ angle. Using the backscatter results from the surface roughness samples, the brighter colors correspond to rougher areas and maybe potential areas of corrosion.

The manufactured sample, shown in figure 10a was approximately $15 \mathrm{~cm}$ by 15 $\mathrm{cm}$. This sample contained four corrosion areas, each $12.7 \mathrm{~mm}$ in diameter, produced by an electrochemical process and four flat bottom holes, $0.15 \mathrm{~mm}$ in depth and $12.7 \mathrm{~mm}$ in diameter. The sample including the corrosion spots and flat bottom holes were painted with a primer and topcoat. This paint layer was $0.06 \mathrm{~mm}$ to $0.07 \mathrm{~mm}$ thick. The flat bottom holes were visible by eye but not the corrosion spots. A $\mathrm{THz}$ amplitude image of the corrosion sample is shown in figure 10b. The amplitude image was generated by taking the minimum signal amplitude between 257 ps and 273 ps. In this image, the darker gray represents greater signal amplitude and the four non-corrosion defects are shown as dark spots and possible detection of the two corrosion defects are in the central area of the plate. The halos around the flat bottom holes are caused by signal interference at the edges, which distort the waveform. This same effect is seen around the outer edges of the sample. In reference to the paint thickness sample, these dark spots would correspond to thicker paint and in reference to the backscatter measurements at normal incidence, the dark areas correspond to increased scattering or rougher areas. In both cases, these spots may indicate potential corrosion.

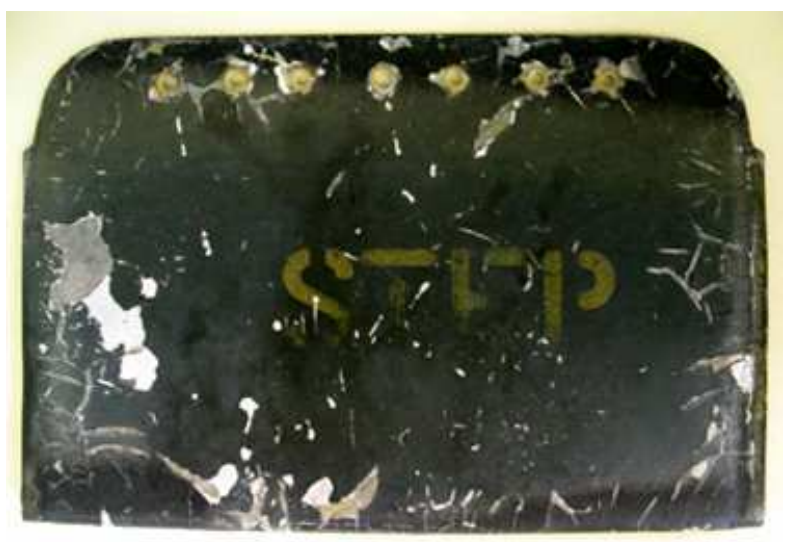

Figure 8. Chinook Fuselage Step Door is shown with flaked, chipped and cracked paint. It is approximately $20 \mathrm{~cm}$ by $14 \mathrm{~cm}$ 


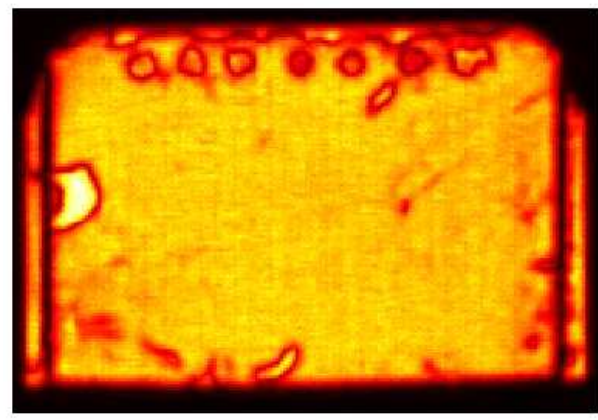

(a)

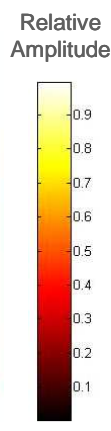

\begin{tabular}{|l}
0.9 \\
-0.8 \\
-0.7 \\
-0.6 \\
0.5 \\
0.4 \\
0.3 \\
0.2 \\
0.1
\end{tabular}

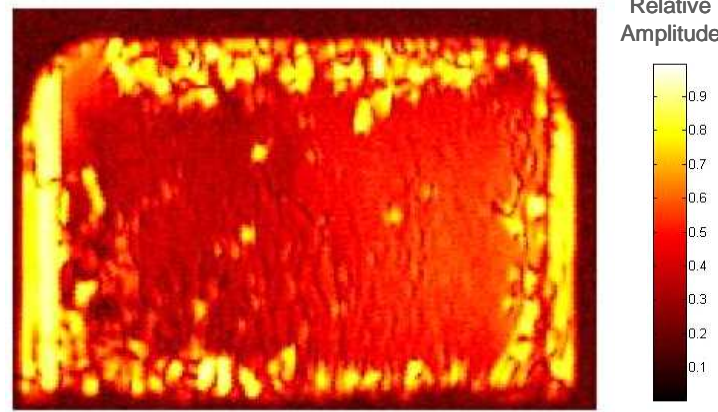

(b)

Figure 9. THz images of the Chinook Fuselage Step Door (a) maximum amplitude, normal inspection and (b) mean frequency amplitude between 0.62 and $0.68 \mathrm{THz}, 10^{\circ}$ angled inspection.

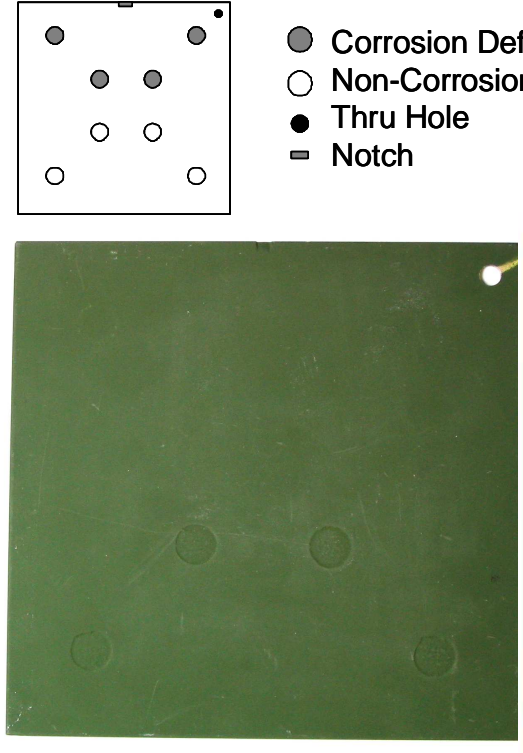

(a)

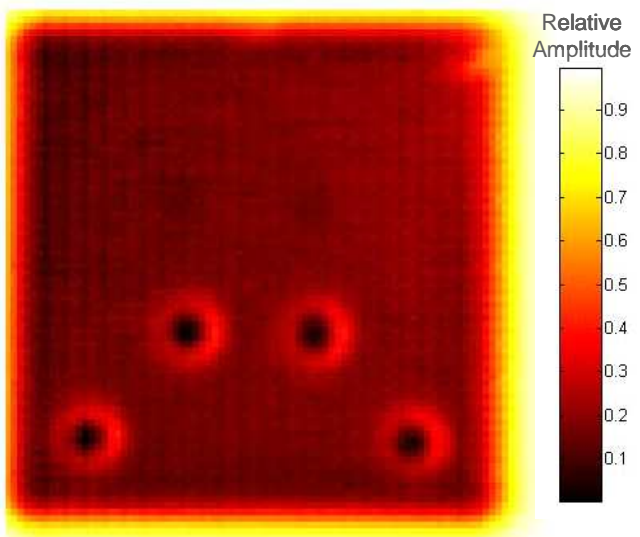

(b)

Figure 10. Corrosion sample (a) picture and (b) THz amplitude image, minimum signal amplitude from 257 ps to 273 ps.

\section{CONCLUSION}

If corrosion under paint causes the paint to swell and increase in thickness or changes a nominally smooth surface to an uneven and irregular surface, then $\mathrm{THz}$ may be able to inspect for corrosion. $\mathrm{THz}$ response to paint thickness showed that as the paint became thin, an individual paint surface echo could not be resolved from substrate echoes, but signal amplitude continued to be influenced by paint thickness. Limits of surface roughness detection were examined using surface-roughness comparator-plates. These plates were scanned and examined in the time and frequency domains and at normal and angled incidence. Surface roughness below $12.5 \mu \mathrm{m}$ Ra became indistinguishable from other surfaces. At normal and low incident angles, backscatter from rough areas was minimal while at higher angles backscatter increased from rough areas and decreased from smoother areas. The frequency images showed this effect better then the signal amplitude 
images. The Chinook Fuselage Step Door may be too complex to characterize without dissecting and studying small sections, but signal amplitude in normal and angled inspection did vary across the sample and may be indications of corrosion or damage. For the manufactured corrosion sample, the dark areas correspond to two corrosion areas and four flat bottom holes. This may have been caused by variations in paint thickness and surface roughness and may be indications of corrosion. Signal processing methods may be able to improve the imaging of these corrosion defects.

\section{REFERENCES}

1. Matzkanin, G.A. and Easter, J.K., "NDE of Hidden Corrosion," Nondestructive Testing and Information Analysis Center, Publication NTIAC-SR-98-03, October 1998.

2. Cargill, J.S., et.al., "Nondestructive Testing for Corrosion Under Paint," Materials Evaluation, Vol. 63, No. 2, February 2005.

3. Hughes, D., et.al., "Detection of Corrosion in Aluminum Panels Under Paint and Primer," Review of Progress in Quantitative Nondestructive Evaluation, Vol. 20, ed by D.O. Thompson and D.E. Chimenti, 2001.

4. Hughes, D., et.al., "Microwave Nondestructive Detection of Corrosion Under Thin Paint and Primer in Aluminum Panels," Surface Sensing Technologies and Applications, Vol. 2, No. 4, October 2001.

5. Mittleman, D., et al., "T-Ray Tomography," Optics Letters, Vol. 22, No. 12, June 1997.

6. Dorney, T., et al., "Imaging with Terahertz Pulses," International Symposium on Optical Science and Technology, July 2000.

7. Hu, B. and Nuss, M.C., "Imaging with Terahertz Waves," Optics Letters, Vol. 20, No. 16, 1995.

8. Mittlemann, D.M., "Recent Advances in Terahertz Imaging," Applied Physics B, 68, 1999.

9. Nemec, H., et al., "Time-Domain Terahertz Study of Defect Formation in OneDimensional Photonic Crystals," Applied Optics, Vol. 43, No. 9, 2004.

10. Pickwell, E., et al., "In Vivo Study of Skin Using Pulsed Terahertz Radiation," Physics in Medicine and Biology, 49, 2004.

11. Precision Devices, Inc, http://www.predev.com/smg/parameters. htm\#Ra\%20\%20Average\%20Roughness, accessed June 2005. 